\title{
Comparison of Harmonic scalpel and monopolar cautery for capsulectomy at the second stage of expander/implant breast reconstruction
}

\author{
Ki Jae Kim, Jae Ho Chung, Hyung Chul Lee, Byung Il Lee, Seung Ha Park, Eul Sik Yoon \\ Department of Plastic and Reconstructive Surgery, Korea University Anam Hospital, Seoul, Korea
}

Background Capsular contracture is a common complication of two-stage expander/implant breast reconstruction. To minimize the risk of this complication, capsulectomy is performed using monopolar cautery or ultrasonic surgical instrumentation, the latter of which can be conducted with a Harmonic scalpel. To date, there is disagreement regarding which of the two methods is superior. The purpose of this study was to compare postoperative outcomes between a group of patients who underwent surgery using a Harmonic scalpel and another group treated with monopolar cautery.

Methods A retrospective chart review was conducted of patients who underwent capsulectomy as part of two-stage breast reconstruction between January 2018 and February 2019 and who received at least 1 month of follow-up after surgery. Operative time and postoperative outcomes, including drainage duration, were analyzed.

Results In total, 36 female patients underwent capsulectomy. The monopolar group consisted of 18 patients and 22 breasts, while the Harmonic scalpel group consisted of 18 patients and 21 breasts. There was no statistically significant difference in demographics between the two groups. The Harmonic scalpel group had a significantly shorter mean drainage duration (6.65 days vs. 7.36 days) and a smaller mean total drainage volume (334.69 mL vs. $433.54 \mathrm{~mL}$ ) than the monopolar cautery group $(P<0.05)$. No statistically significant difference was observed with regard to seroma or hematoma formation.

Conclusions The Harmonic scalpel approach for capsulectomy reduced the total drainage volume and drainage duration compared to the monopolar cautery approach. Therefore, this approach could serve as a good alternative to electrocautery.

Keywords Breast / Capsules / Ultrasonic vibration / Seroma
Correspondence: Eul Sik Yoon Department of Plastic and Reconstructive Surgery, Korea University Anam Hospital, 73 Inchonro, Seongbuk-gu, Seoul 02841, Korea Tel: $+82-2-920-5368$

Fax: +82-2-920-7437

E-mail: yesanam2@korea.ac.kr

Received: August 27, 2019 • Revised: January 7, 2020 • Accepted: January 14, 2020

pISSN: 2234-6163 • elSSN: 2234-6171 • https://doi.org/10.5999/aps.2019.01130 • Arch Plast Surg 2020;47:140-145

\section{INTRODUCTION}

Breast cancer is the most frequently-occurring cancer in women and is the second most frequently-occurring cancer, after only lung cancer, overall. Breast reconstruction has become an inte- gral part of treatment due to long-term psychosexual and aesthetic health factors. The major reconstructive options are autologous tissue-based reconstruction and implant-based reconstruction. Of these, implant-based reconstruction is the more frequently performed procedure in the United States, South 
Korea, and other countries, and its utilization continues to increase. This trend has led to a thorough consideration of the risks and complications associated with silicone implants [1].

Capsular contracture is the most common and frustrating complication of breast implantation for patients. In large studies, the overall incidence of capsular contracture after silicone implant insertion has been reported to range from $4 \%$ to $17 \%$ [24]. Traditionally, capsular contracture is treated surgically. Currently, the gold standard for treatment is capsulectomy with or without capsulotomy.

A common technique used in capsulectomy is monopolar cautery, which is used to electrically damage tissue using current administered from a metal electrode. However, the substantial thermal spread associated with this technique can potentially promote flap necrosis and infection. Moreover, the use of monopolar cautery may elevate the risk for increased drainage volume and seroma formation via heat injury to the nearby lymphatic vessels.

Ultrasonic surgical instrumentation (Harmonic scalpel; Ethicon, Somerville, NJ, USA) was introduced into the clinical field to mitigate the risks of traditional electrosurgery, including damage to nearby areas and potentially catastrophic complications - such as seroma - related to monopolar energy delivery. Harmonic scalpels use $55.5 \mathrm{kHz}$ ultrasonic vibrations and a vibratory excursion of 50-100 $\mu \mathrm{m}$ to denature proteins; these proteins then aggregate in the vessel lumen, coapting and sealing the vessel. The temperature is lower than that involved in other techniques, this minimizes thermal injury, especially to lymphatic vessels, and notably reduces the rate of postoperative complications [5].

However, no study to date has compared the effects of the Harmonic scalpel and monopolar cautery in capsulectomy, although several studies have compared them in mastectomy. One previous study reported no significant difference in intraoperative bleeding, operative time, or postoperative wound complications between the two methods when used for total mastectomy [6]. However, Anandaravi et al. [7] compared the Harmonic scalpel with monopolar cautery in modified radical mastectomy in 2018 and found that the Harmonic scalpel group had smaller drainage volumes and less blood loss during surgery than the monopolar cautery group. Currently, the difference in efficacy between the Harmonic scalpel and monopolar cautery remains uncertain, especially in capsulectomy.

Therefore, the goal of this study was to compare the postoperative outcomes of capsulectomy performed using the Harmonic scalpel and monopolar cautery.

\section{METHODS}

A retrospective review was conducted of patients who presented at Korea University Anam Hospital for two-stage breast reconstruction between January 2018 and February 2019. The study design was approved by the Committee for the Protection of Human Subjects at Korea University Medical Center in Seoul (IRB No. 2019AN0476). Patients were included if they underwent replacement with a permanent implant followed by capsulectomy using monopolar cautery or a Harmonic scalpel (HD 1000i Shears handpiece, curved tip, and GEN11 generator; Ethicon) in the second stage of breast reconstruction (Supplemental Video 1). In all cases, smooth implants manufactured by BellaGel (Hans Biomed Corp., Seoul, Korea), Mentor (Santa Barbara, CA, USA), or Motiva (Establishment Labs; Alajuela, Costa Rica) were used. The method of choice at our institution was partial capsulectomy. In most instances of this procedure, a marginal strip of capsule was removed. In cases where the adhesion between the capsule and the adjacent breast tissue was relatively weak, total capsulectomy was performed instead. All operations were performed by a single surgeon (ESY). Any patient with a follow-up period of less than 1 month was excluded from this study.

Patient demographic and clinical data, including information regarding post-mastectomy therapy, were collected. Operative time and postoperative outcomes, including drainage duration, were also collected. Whenever the drainage amount was less than $20 \mathrm{~mL}$ for two consecutive days, the drain was removed. In this study, seroma was defined as any subcutaneous fluid collection after drain removal with aspiration of a volume greater than $50 \mathrm{~mL}$.

All statistical analyses were performed using SPSS version 23 (IBM Corp., Armonk, NY, USA). The demographic information and operative outcomes of the two groups (Harmonic scalpel vs. monopolar cautery) were compared using the paired $t$ test. P-values less than 0.05 were considered to indicate statistical significance.

\section{RESULTS}

Data from 36 female patients who had undergone capsulectomy in the second stage of breast reconstruction were reviewed. Eighteen patients ( 22 breasts) underwent surgery with monopolar cautery, and 18 patients ( 21 breasts) underwent surgery with a Harmonic scalpel. In the monopolar group, the mean age was 49.09 years, and the mean body mass index (BMI) was $22.44 \mathrm{~kg} / \mathrm{m}^{2}$. In the Harmonic scalpel group, the mean age and mean BMI were 49.71 years and $22.85 \mathrm{~kg} / \mathrm{m}^{2}$, respectively. 
There was no significant difference in age or BMI between the two groups, and there were no smokers in either group.

The mean volume of the permanent implant inserted during second-stage surgery was $314.19 \mathrm{~mL}$. There was no significant difference in permanent implant volume between the groups $(316.82 \mathrm{~mL}$ in the monopolar cautery group vs. $310.88 \mathrm{~mL}$ in the Harmonic scalpel group; $\mathrm{P}=0.852$ ). In all cases, smooth implants were used. In the Harmonic scalpel group, 18 implants were manufactured by BellaGel, two by Mentor, and one by Motiva. In the monopolar cautery group, 16 implants were manufactured by BellaGel and six by Mentor.

There was no statistically significant difference in perioperative adjuvant therapy between the two groups. Chemotherapy between first- and second-stage surgery was more frequent in the Harmonic scalpel group (nine breasts) than in the monopolar cautery group (six breasts). However, the difference was not statistically significant $(P=0.108)$. Similarly, no statistically significant difference was observed in the interval between firstand second-stage surgery $(\mathrm{P}=0.533)$ (Table 1).

\begin{tabular}{|c|c|c|c|}
\hline Characteristic & $\begin{array}{l}\text { Monopolar } \\
\text { cautery group } \\
(n=22)\end{array}$ & $\begin{array}{l}\text { Harmonic } \\
\text { scalpel group } \\
(n=21)\end{array}$ & P-value \\
\hline Age (yr) & 49.09 & 49.71 & 0.825 \\
\hline BMl $\left(\mathrm{kg} / \mathrm{m}^{2}\right)$ & 22.44 & 22.85 & 0.722 \\
\hline Implant size (mL) & 316.82 & 310.88 & 0.852 \\
\hline \multicolumn{4}{|l|}{ Therapy, no. } \\
\hline Hormone & 13 & 12 & 0.471 \\
\hline Chemotherapy & 6 & 9 & 0.108 \\
\hline Radiation & 3 & 2 & 0.867 \\
\hline Capsulectomy, no. & & & 0.739 \\
\hline Partial & 16 & 17 & \\
\hline Total & 6 & 4 & \\
\hline $\begin{array}{l}\text { Period between first and } \\
\text { second stage (mon) }\end{array}$ & 5.66 & 5.42 & 0.533 \\
\hline
\end{tabular}

Table 2. Outcome comparison between the monopolar cautery and the Harmonic scalpel groups

\begin{tabular}{|lccc|}
\hline Outcome & $\begin{array}{c}\text { Monopolar } \\
\text { cautery group } \\
(\mathbf{n = 2 2})\end{array}$ & $\begin{array}{c}\text { Harmonic } \\
\text { scalpel group } \\
(\mathbf{n}=\mathbf{2 1})\end{array}$ & P-value \\
\hline Operative time (min) & 172.95 & 149.41 & 0.337 \\
Hospital stay duration (day) & 8.64 & 8.71 & 0.110 \\
Drainage duration (day) & 7.36 & 6.65 & $0.047^{\text {a) }}$ \\
Drainage volume (mL) & 433.54 & 334.69 & $0.027^{\text {a) }}$ \\
\hline $\begin{array}{l}\text { Values are presented as mean. } \\
\text { a)P-values < } 0.05 \text { indicate statistical significance. }\end{array}$ \\
\hline
\end{tabular}

In the Harmonic scalpel group, partial capsulectomy was performed in 17 cases and total capsulectomy in four cases. In the monopolar cautery group, partial capsulectomy was performed in 16 cases and total capsulectomy in six cases.

No significant difference in operative time or hospital stay duration was found between the two groups. However, the Harmonic scalpel group had a significantly shorter mean drainage duration ( 6.65 days vs. 7.36 days, $\mathrm{P}=0.047$ ) and a smaller mean total drainage volume (334.69 mL vs. $433.54 \mathrm{~mL}, \mathrm{P}=0.027)$ than the monopolar cautery group (Table 2).

In addition, the drainage volumes of the groups were compared daily. Fig. 1 shows the daily accumulated drainage volume for the two groups; the difference between groups increased over time.

Other postoperative complications, including seroma, hematoma, and skin flap necrosis, were monitored. There were no significant differences in the rates of these complications between the two groups. Seroma was found in two breasts in the monopolar group, but was not observed in the Harmonic group,

\section{Fig. 1. Daily comparison of drainage volume}

Drainage volume was compared daily between the monopolar cautery group and the Harmonic scalpel group. The difference in the total accumulation of drainage between the two groups increased over time.

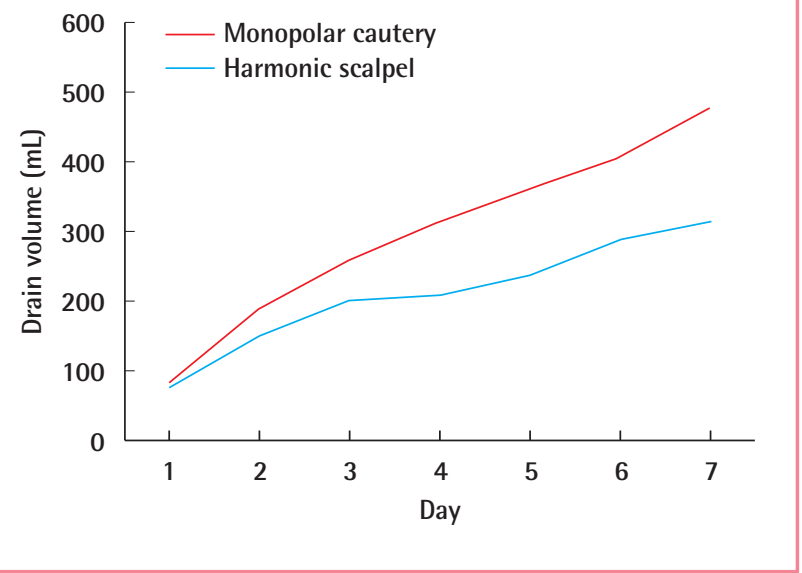

Table 3. Complications in the monopolar cautery and the Harmonic scalpel groups

\begin{tabular}{|lccc|}
\hline Complication & $\begin{array}{c}\text { Monopolar } \\
\text { cautery group } \\
(\mathbf{n}=\mathbf{2 2})\end{array}$ & $\begin{array}{c}\text { Harmonic } \\
\text { scalpel group } \\
(\mathbf{n}=\mathbf{2 1})\end{array}$ & P-value \\
\hline Seroma & $2(9.5)$ & 0 & 0.212 \\
Hematoma & 0 & $1(16.7)$ & 0.261 \\
Skin flap necrosis & 0 & 0 & NA \\
\hline $\begin{array}{l}\text { Values are presented as number (\%). } \\
\text { NA, not available. }\end{array}$ & & \\
\hline
\end{tabular}


constituting no significant difference between groups. Hematoma occurred in one breast in the Harmonic group but did not occur in the monopolar group, similarly constituting no significant difference. Skin flap necrosis was not found in either group (Table 3).

\section{DISCUSSION}

Seromas frequently arise due to tissue disruption and dissection that leads to the presence of dead space and disrupted lymphatic flow, which can occur as a result of lymph node dissection, mastectomy, or capsulectomy. The Harmonic scalpel uses ultrasonic waves that disrupt the hydrogen bonds of proteins within the tissue, leading to denaturation. The denatured proteins then interact with intracellular and interstitial fluids, forming a glue-like coagulum that can seal off vessels and lymphatics, which in turn leads to decreases in blood loss and lymphatic leakage. This disruption occurs at a lower temperature than is the case for both electrocautery and laser energy, thus causing fewer tissue burns $[8]$.

Previous studies have reported postoperative seroma formation after mastectomy. Some of these studies showed similar results to ours, even though they focused on modified radical mastectomy [7,9-11].

Anandaravi et al. [7] studied the efficacy of Harmonic scalpel usage over electrocautery in modified radical mastectomy. Their results showed that the use of the Harmonic scalpel approach decreased the total operating time, the axillary dissection time, the amount of blood loss, the total drainage volume, and the number of days of drainage. Mittal et al. [6] also compared the Harmonic scalpel and monopolar cautery in modified radical mastectomy. They showed that use of the Harmonic scalpel resulted in smaller drainage volumes and less intraoperative blood loss than monopolar cautery. However, no statistical difference was observed in drain duration or in the occurrence of seroma, hematoma, or flap necrosis between the two groups. Chang et al. [10] compared the LigaSure scalpel (Covidien, Boulder, CO, USA) with electrocautery in skin-sparing mastectomy. LigaSure is a device similar to the Harmonic scalpel, and it provides hemostasis via the use of electrothermal energy and applied pressure to establish a seal. Chang et al. found that the LigaSure group had a smaller total drainage volume and fewer days of drainage than the electrocautery group.

In our study, both drainage duration and total drainage volume were significantly lower in the group of patients operated upon with the Harmonic scalpel, which is consistent with the studies previously mentioned. However, those studies focused on modified radical mastectomy. To the best of our knowledge, our study is the first to compare the Harmonic scalpel with electrocautery in capsulectomy.

Drain placement is intended to reduce complications, including hematoma, seroma, and flap necrosis secondary to seroma. Electrocautery can promote imprecise hemostasis and lymphostasis; additionally, it can lead to increased morbidity through the severing of and thermal injury to lymphatic vessels and through hematoma formation. In comparison, the Harmonic scalpel generates ultrasonic waves that break hydrogen bonds. This produces a coagulated mass of denatured proteins, sealing blood and lymphatic vessels and decreasing both the loss of blood and lymphatic drainage. Inflammation in the operative field is similarly decreased, as is the damage to lymphatic tissue and the extent of oozing in the area of the operation. Together, these phenomena are believed to contribute to the reduced drainage volume observed in the Harmonic scalpel group in our study. Based on this biological explanation, seroma or hematoma occurrence is expected to be more frequent in the electrocautery group than in the Harmonic scalpel group. However, in our study, there was no statistically significant difference in seroma or hematoma occurrence between the two groups. The studies mentioned above also found no statistically significant differences in seroma or hematoma formation.

Our findings may be attributable to our small sample size. In our study, a total of 33 patients were enrolled, similar to the 50 or fewer patients enrolled in previous studies. A larger patient group might uncover differences in the rates of seroma or hematoma occurrence between the electrocautery and Harmonic scalpel groups.

The rate of flap necrosis can be decreased by using energy-administering devices such as Harmonic or LigaSure scalpels in conventional total mastectomy [12-15]. According to previous studies, flap necrosis is related to lateral thermal spread from the surgical device. In one report, Sutton et al. [15] demonstrated that, of the electrosurgical techniques assessed in their study, electrocautery was associated with the largest degree of thermal spread and the highest average temperature. Although our results indicated no significant difference in the rate of flap necrosis between the two groups, additional studies with more subjects are needed to further elucidate the factors involved in flap necrosis.

Lastly, we must take into account a cost-benefit analysis between the two groups. The greatest disadvantage of the Harmonic scalpel is its relatively high financial cost compared to electrocautery. According to a prior study by Burdette et al. [16], per case cost was notably higher for the disposable elements of the Harmonic scalpel (\$275 per case) than for those of the electrocautery device ( $\$ 11.45$ per case). However, this cost can be 
offset by the reduced operation time and drainage volume. Therefore, further studies are necessary to outline a cost-benefit analysis that takes into account these factors. We believe that the cost of using the Harmonic scalpel is acceptable for patients given its advantages regarding postoperative outcomes.

There were some limitations to this study, including its retrospective nature, which introduces the possibility of bias. The differing numbers of breasts in the two groups, as well as the small number of subjects, were also limitations of the study. Despite these limitations, the results have clinical significance in that they provide insights into the Harmonic scalpel approach for capsulectomy.

In conclusion, the utilization of the Harmonic scalpel during capsulectomy was associated with a lower total drainage volume and a shorter drainage duration than those observed after monopolar cautery. Other parameters, including the occurrence of hematoma, seroma, or flap necrosis, were not significantly different between the two study groups. Further studies are needed to confirm these findings. However, based on these results, the Harmonic scalpel is potentially a good alternative for electrocautery, particularly given its beneficial effects on drainage.

\section{NOTES}

\section{Conflict of interest}

No potential conflict of interest relevant to this article was reported.

\section{Ethical approval}

The study was approved by the Institutional Review Board of Korea University Medical Center (IRB No. 2019AN0476) and performed in accordance with the principles of the Declaration of Helsinki.

\section{Author contribution}

Study concept and design: Yoon ES. Data analysis and interpretation: Kim KJ, Chung JH, Lee HC. Drafting of the manuscript: Kim KJ, Chung JH, Lee HC. Critical revision of the manuscript for intellectual content: Park SH, Lee BI, Yoon ES. Approval of final manuscript: all authors.

\section{ORCID}

KiJae Kim

Jae Ho Chung

Hyung Chul Lee

Byung Il Lee

Seung Ha Park

Eul Sik Yoon https://orcid.org/0000-0002-0979-6688

https://orcid.org/0000-0002-8351-2444

https://orcid.org/0000-0003-2482-8112

https://orcid.org/0000-0001-8183-0388

https://orcid.org/0000-0002-2667-2791

https://orcid.org/0000-0001-5734-6625

\section{Supplementary material}

Supplemental Video 1. Capsulectomy using Harmonic scalpel. Supplemental data can be found at: https://doi.org/10.5999/ aps.2019.01130.v001

\section{REFERENCES}

1. Reyna C, Lee MC. Breast cancer in young women: special considerations in multidisciplinary care. J Multidiscip Healthc 2014;7:419-29.

2. Handel N, Jensen JA, Black $Q_{2}$ et al. The fate of breast implants: a critical analysis of complications and outcomes. Plast Reconstr Surg 1995;96:1521-33.

3. Henriksen TF, Fryzek JP, Holmich LR, et al. Surgical intervention and capsular contracture after breast augmentation: a prospective study of risk factors. Ann Plast Surg 2005;54: 343-51.

4. Caffee HH. Textured silicone and capsule contracture. Ann Plast Surg 1990;24:197-9.

5. Foschi D, Cellerino P, Corsi F, et al. The mechanisms of blood vessel closure in humans by the application of ultrasonic energy. Surg Endosc 2002;16:814-9.

6. Mittal P, Kumar A, Kaur S, et al. A comparative study of the use of harmonic scalpel versus unipolar cautery in modified radical mastectomy. Niger J Surg 2017;23:20-5.

7. Anandaravi BN, Parveen PN, Mohammed AA. A study showing efficacy of harmonic scalpel over electrocautery in modified radical mastectomy. Int Surg J 2017;4:1422-5.

8. Sharma AK, Kumar M, Singh A, et al. A prospective randomized study comparing ultrasonic dissector with monopolar electrocautery for dissection in modified radical mastectomy. Int J Surg Sci 2019;3:149-53.

9. Alptekin H, Yilmaz H, Ozturk B, et al. Comparison of electrocautery and plasmablade on ischemia and seroma formation after modified radical mastectomy for locally advanced breast cancer. Surg Tech Dev 2017;7:7011.

10. Chang Y, Kim H, Jung S, et al. Comparison of skin-sparing mastectomy using LigaSure ${ }^{\mathrm{TM}}$ Small Jaw and electrocautery. World J Surg Onc 2017; 15:129.

11. Khan S, Khan S, Chawla T, et al. Harmonic scalpel versus electrocautery dissection in modified radical mastectomy: a randomized controlled trial. Ann Surg Oncol 2014;21:80814.

12. Kiyingi AK, Macdonald LJ, Shugg SA, et al. Harmonic dissection versus electrocautery in breast surgery in regional Victoria. ANZJ Surg 2015;85:358-62.

13. Anlar B, Karaman N, Dogan L, et al. The effect of harmonic scalpel, electrocautery, and scalpel use on early wound com- 
plications after modified radical mastectomy. Eur Surg 2013; 45:286-90.

14. Galatius H, Okholm M, Hoffmann J. Mastectomy using ultrasonic dissection: effect on seroma formation. Breast 2003; 12:338-41.

15. Sutton PA, Awad S, Perkins AC, et al. Comparison of lateral thermal spread using monopolar and bipolar diathermy, the Harmonic Scalpel and the Ligasure. Br J Surg 2010;97:42833.

16. Burdette TE, Kerrigan CL, Homa K. Harmonic scalpel versus electrocautery in breast reduction surgery: a randomized controlled trial. Plast Reconstr Surg 2011;128:243e-249e. 